\title{
Chromospheric evaporation flows and density changes deduced from Hinode/EIS during an M1.6 flare ${ }^{\star}$
}

\author{
P. Gömöry ${ }^{1}$, A. M. Veronig ${ }^{2}, \mathrm{Y} \mathrm{Su}^{2,3}, \mathrm{M}$. Temmer ${ }^{2}$, and J. K. Thalmann ${ }^{2}$ \\ ${ }^{1}$ Astronomical Institute of the Slovak Academy of Sciences, 05960 Tatranská Lomnica, Slovakia \\ e-mail: gomory@astro.sk \\ 2 IGAM-Kanzelhöhe Observatory, Institute of Physics, University of Graz, Universitätsplatz 5, 8010 Graz, Austria \\ 3 Key Laboratory of Dark Matter \& Space Astronomy, Purple Mountain Observatory, Chinese Academy of Sciences, \\ 2 West Beijing Road, 210008 Nanjing, PR China
}

Received 18 September 2015 / Accepted 26 January 2016

\begin{abstract}
Aims. We study the response of the solar atmosphere during a GOES M1.6 flare using spectroscopic and imaging observations. In particular, we examine the evolution of the mass flows and electron density together with the energy input derived from hard X-ray (HXR) in the context of chromospheric evaporation.

Methods. We analyzed high-cadence sit-and-stare observations acquired with the Hinode/EIS spectrometer in the Fe XIII $202.044 \AA(\log T=6.2)$ and Fe XVI $262.980 \AA(\log T=6.4)$ spectral lines to derive temporal variations of the line intensity, Doppler shifts, and electron density during the flare. We combined these data with HXR measurements acquired with RHESSI to derive the energy input to the lower atmosphere by flare-accelerated electrons.

Results. During the flare impulsive phase, we observe no significant flows in the cooler Fe XIII line but strong upflows, up to $80-150 \mathrm{~km} \mathrm{~s}^{-1}$, in the hotter Fe XVI line. The largest Doppler shifts observed in the Fe XVI line were co-temporal with the sharp intensity peak. The electron density obtained from a Fe XIII line pair ratio exhibited fast increase (within two minutes) from the pre-flare level of $5.01 \times 10^{9} \mathrm{~cm}^{-3}$ to $3.16 \times 10^{10} \mathrm{~cm}^{-3}$ during the flare peak. The nonthermal energy flux density deposited from the coronal acceleration site to the lower atmospheric layers during the flare peak was found to be $1.34 \times 10^{10} \mathrm{erg} \mathrm{s}^{-1} \mathrm{~cm}^{-2}$ for a low-energy cut-off that was estimated to be $16 \mathrm{keV}$. During the decline flare phase, we found a secondary intensity and density peak of lower amplitude that was preceded by upflows of $\sim 15 \mathrm{~km} \mathrm{~s}^{-1}$ that were detected in both lines. The flare was also accompanied by a filament eruption that was partly captured by the EIS observations. We derived Doppler velocities of $250-300 \mathrm{~km} \mathrm{~s}^{-1}$ for the upflowing filament material.

Conclusions. The spectroscopic results for the flare peak are consistent with the scenario of explosive chromospheric evaporation, although a comparatively low value of the nonthermal energy flux density was determined for this phase of the flare. This outcome is discussed in the context of recent hydrodynamic simulations. It provides observational evidence that the response of the atmospheric plasma strongly depends on the properties of the electron beams responsible for the heating, in particular the steepness of the energy distribution. The secondary peak of line intensity and electron density detected during the decline phase is interpreted as a signature of flare loops being filled by expanding hot material that is due to chromospheric evaporation.
\end{abstract}

Key words. Sun: flares - Sun: chromosphere - Sun: corona - Sun: UV radiation

\section{Introduction}

Flares are among the most energetic events on the Sun. According to the standard eruptive flare model (Carmichael 1964; Sturrock 1968; Hirayama 1974; Kopp \& Pneuman 1976; see also reviews by Benz 2008; Fletcher et al. 2011, and references therein), the energy to power solar flares is stored in the nonpotential coronal magnetic fields (e.g., Forbes 2000; Hudson 2011; Wiegelmann et al. 2014). The mechanism that releases magnetic energy is known as magnetic reconnection. Observational as well as theoretical aspects of this mechanism have been studied in detail (e.g., Parker 1963; Demoulin et al. 1993; Litvinenko \& Somov 1993; Yokoyama et al. 2001; Aulanier et al. 2006; Veronig et al. 2006; Su et al. 2013; Dudík et al. 2014; van Driel-Gesztelyi et al. 2014; Li \& Zhang 2015).

The vast amount of energy released during magnetic reconnetion in the corona is converted into heating of the surrounding plasma and accelerating particles to nonthermal energies. The

\footnotetext{
* A movie is available at http://www . aanda. org
}

flare-accelerated particles are guided by the ambient magnetic field and progress downward to the lower atmosphere, encountering a denser environment. They are effectively stopped at chromospheric heights where their energy is dissipated by Coulomb collisions with the ambient thermal particles (Brown 1971; Lin \& Hudson 1976). This rapid energy deposition causes the chromospheric plasma to be intensely heated to coronal and flare $\left(10^{7} \mathrm{~K}\right)$ temperatures, expanding upward, and thus to fill the coronal loops. This process is called chromospheric evaporation and was first proposed by Neupert (1968) to explain the observed correlation between the thermal and integrated nonthermal flare emission (e.g., Veronig et al. 2002).

The process of chromospheric evaporation was investigated in a number of theoretical studies. Hydrodynamic simulations revealed two evaporation regimes that are separated by an energy flux density threshold at roughly $10^{10} \mathrm{erg} \mathrm{cm}^{-2} \mathrm{~s}^{-1}$ (e.g., Fisher et al. 1985). So-called gentle evaporation occurs if the energy that is deposited in the chromosphere by nonthermal electrons is lower than this threshold. The heated chromospheric material then slowly expands upward with velocities of several tens 
of $\mathrm{km} \mathrm{s}^{-1}$. In addition, gentle evaporation may be also a result of thermal conduction from the hot flaring corona. Signatures of gentle evaporation were observed mainly during weaker flares (i.e., GOES C-class and weaker) as well as during the preflare and late phase of stronger events (Czaykowska et al. 1999; Brosius \& Phillips 2004; Milligan et al. 2006b).

If the energy flux densities deposited in the chromosphere exceed $10^{10} \mathrm{erg} \mathrm{cm}^{-2} \mathrm{~s}^{-1}$, explosive evaporation takes place. In this case, the radiative cooling is insufficient and the plasma is rapidly heated to coronal and flare $\left(10^{7} \mathrm{~K}\right)$ temperatures. As a consequence, the local gas pressure rises significantly, yielding an explosive upward expansion of the chromospheric plasma at velocities reaching several hundred $\mathrm{km} \mathrm{s}^{-1}$. To regain momentum balance with the hot plasma upflows, the cooler material of the underlying layers is pushed downward. Simulations predict that downflows are noticeable only in spectral lines formed below transition region temperatures and that they reach velocities of several tens of $\mathrm{km} \mathrm{s}^{-1}$ because of the higher mass and inertia of the chromospheric material (Fisher et al. 1985; Allred et al. 2005; Reep et al. 2015). Observational evidence for the proposed momentum balance during explosive evaporation was found for several events (Zarro et al. 1988; Teriaca et al. 2006; Milligan et al. 2006a). Other observational studies, however, also revealed downflows at coronal temperatures (Milligan \& Dennis 2009; Young et al. 2013), suggesting that the flaring atmosphere is very dynamic and complex on small spatial scales (e.g., Veronig et al. 2010). Simplified hydrodynamic simulations therefore probably fall short of adequately describing all responses of the flaring atmosphere.

In general, a characteristic signature of chromospheric evaporation are observed spectral blueshifts during the impulsive phase of flares as the consequence of heated plasma rising into the corona. This has been observed more than 30 years ago (Doschek et al. 1980; Feldman et al. 1980; Antonucci et al. 1982). However, one-dimensional simulations of chromospheric evaporation in a single flare loop predict a blueshift of the spectral line profiles as a whole during the early phases of flares (Emslie \& Alexander 1987; McClements \& Alexander 1989). This contradicts the majority of the earliest observational findings, which only revealed an asymmetry in the blue wing of the spectral line profiles (commonly interpreted as evidence of upflows of several hundred $\mathrm{km} \mathrm{s}^{-1}$ ), together with a dominant static spectral component (e.g., Antonucci et al. 1999). This obvious discrepancy is solved when a multi-thread, fine-structured flare loop model is considered (Hori et al. 1997; Doschek \& Warren 2005; Warren \& Doschek 2005). In this case, the asymmetric line profiles are the result of superposed spectral components at different velocities that are emitted from particular threads within the flare loop envelope. We stress, however, that recent results based on high-resolution measurements clearly demonstrate the existence of entirely blueshifted spectral profiles during the onset phases of flares (Del Zanna et al. 2006, 2011; Brosius 2013a; Polito et al. 2015).

Another aspect of the chromospheric evaporation process is the enhancement of the electron density measured at high temperatures. Electron densities are often determined based on methods that use density-sensitive line ratios under the assumption that one of these lines arises from a meta-stable level (for more details see, e.g., Mariska 1992). It is important to determine them accurately to understand the heating and cooling of flaring plasma. Time-dependent studies of the density variations during flares are quite rare. One of the first results were published by McKenzie et al. (1980) and Doschek et al. (1981), who used data from the SOLEX instrument onboard P78-1.
Graham et al. (2011) used EIS observations to study the temporal evolution of densities during a C6.6 flare, but with a limited time-resolution of $150 \mathrm{~s}$. Recently, Milligan et al. (2012) presented techniques for determining time-dependent measurements of the electron density using high-temperature $(\sim 12 \mathrm{MK})$ density-sensitive line pairs measured with the SDO/EVE instrument. They found that the most reliable line pairs detected by the EVE full-Sun measurements are sensitive to densities in the range $10^{11}-10^{14} \mathrm{~cm}^{-3}$ and are therefore suitable to study density variations during $\mathrm{X}$-class flares, where such enhancements of electron densities may develop. However, electron densities exceeding $10^{11} \mathrm{~cm}^{-3}$ (i.e., the low-density limit for the EVE measurements) are rather unrealistic for M-class flares.

In general, flare spectroscopy can be performed in two ways: raster and sit-and-stare observations. Most of the existing studies are based on rasters. This observing mode provides good insight into the spatial distribution of the response of the flaring atmosphere, but the data are acquired only with very limited temporal resolution (order of minutes) for dynamic events like flares. Therefore, the locations of the main energy deposition (flare kernels) are often missed in raster scans, as they have only short lifetimes. To study the temporal evolution of the plasma properties in flare kernels, sit-and-stare observations are preferable. Since the slit covers only a very limited and fixed field of view, it is difficult to obtain data across entire flare kernels, however. Thus, flare studies based on sit-and-stare observations are still rare (e.g., Brosius 2001, 2013a; Berkebile-Stoiser et al. 2009; Veronig et al. 2010; Brosius \& Daw 2015).

We here present a study on the chromospheric evaporation process during an M-class flare using one such rare data set of sit-and-stare measurements from Hinode/EIS, where the slit was placed across a flare kernel. We focus on the flareinduced Doppler velocities and densities acquired with high cadence. The spectroscopic findings are discussed in the context of high-cadence EUV imaging by AIA/SDO and the characteristics of the flare-accelerated electrons deduced from RHESSI hard X-ray images and spectra.

\section{Data and data reduction}

The data-set presented here was obtained within the Hinode Observing Plan HOP- $180^{1}$ that was performed during several days in February 2011. This observing program was specifically designed to study spectroscopic properties and dynamics of large-scale coronal waves (so-called EIT waves) propagating through the quiet corona by combining high-cadence spectroscopy with high-cadence multiwavelength imaging (Veronig et al. 2011). The main observing target was AR 11158, which became the first major flaring region of solar cycle 24 . The magnetic structure and evolution of this active region as well as the properties of the $\mathrm{M}$ - and X-class flares it produced have been studied in a number of papers (e.g., Young et al. 2011, 2013; Kosovichev 2011; Schrijver et al. 2011; Sun et al. 2012; Inoue et al. 2013; Sorriso-Valvo et al. 2015; Inoue et al. 2015; Kuroda et al. 2015).

We concentrate on the analysis of a subset of the data obtained on 16 February 2011 between 13:38 and 15:43 UT. This subset covers the evolution of an eruptive M1.6 flare that was accompanied by an EIT wave. The properties and plasma diagnostics of the EIT wave have been presented in Harra et al. (2011), Veronig et al. (2011), and Long et al. (2013).

\footnotetext{
1 http://www . isas.jaxa.jp/home/solar/hinode_op/hop. php?hop $=0180$
} 
The spectroscopic data were acquired with the EUV Imaging Spectrometer (EIS; Culhane et al. 2007) onboard the Japanese space mission Hinode (Kosugi et al. 2007). The longest possible slit with height $512^{\prime \prime}$ and width $2^{\prime \prime}$ (with a pixel size of $1^{\prime \prime}$ in the $y$-direction) was used in the sit-and-stare observing mode. The exposure time was set to $45 \mathrm{~s}$ plus $\sim 4 \mathrm{~s}$ for read out. The effective time cadence is thus $\sim 49 \mathrm{~s}$. The standard runs of HOP-180 were performed using 11 selected spectral lines that cover the temperature range $\log T=4.7-6.7$, including several line pairs from the same ion for density diagnostics. However, several of these lines are unsuitable for the flare study. The main goal of HOP-180 was the detection of EIT waves in the quiet solar corona, and therefore blended lines were also selected because the blends are very weak under conditions of weak intensity enhancements. But they become significant in flaring regions.

Multi-Gaussian fitting can in principle be used to separate such profiles, but this method must be applied very carefully as it is susceptible to creating statistically acceptable but unphysical fits to the data. Therefore it is normally applied to single spectra where the result can be verified, but it is not very suitable to fit longer time-series. We therefore concentrate here on the spectral lines with no blends and a strong signal: Fe XIII $202.044 \AA(\log T=6.2)$ and Fe XVI $262.980 \AA(\log T=6.4)$. In addition, we used the Fe XIII $196.640 \AA$ spectral line to determine coronal electron densities from the Fe XIII line pair. The electron densities were estimated using the theoretical variation of the line intensity ratio with density using the CHIANTI database version 7.1. (Dere et al. 1997; Landi et al. 2013). After the theoretical ratio was known, the final density maps were calculated using the EIS routine eisdensity.pro.

The EIS data were first corrected for photometric effects and calibrated using the eis_prep.pro routine, which is part of the SolarSoftWare. The wavelength drift was compensated for using the so-called HK method described in Kamio et al. (2010). Then the spectral profiles were fit by a single-Gaussian function with a linear background to obtain the amplitude of the profile, integrated intensities, background intensities, Doppler shifts, and spectral widths. The spatial offset in the solar $y$-direction among the data detected at different wavelengths was also compensated for. Because EIS provides no absolute wavelength scale, the determination of a rest wavelength is difficult especially in active regions. In our case, the longest slit that also covered quiet areas was used, and the zero reference of the Doppler shifts was calculated as the average value of the Doppler shifts from quiet-Sun regions. We adopted the formalism that positive velocities (redshifts) represent motions toward the solar surface and negative velocities (blueshifts) denote motions into the corona.

The imaging was performed using the Atmospheric Imaging Assembly (AIA; Lemen et al. 2012), which is part of the Solar Dynamics Observatory (SDO; Pesnell et al. 2012). AIA carries four telescopes and obtains full-Sun images at a $12 \mathrm{~s}$ cadence in seven different EUV filters and at a $24 \mathrm{~s}$ cadence in two $U V$ filters. The spatial resolution is $\sim 1$.'5 with a corresponding pixel size of 0 '. $^{\prime} 6 \times 0 \times 0^{\prime} 6$. We here in particular used data taken in the AIA $304 \AA(\log T=4.7), 171 \AA(\log T=5.8)$, and $94 \AA(\log T=6.8)$ channels. The AIA data were downloaded from the Virtual Solar Observatory (VSO) in the level-1 format, that is, they were already corrected for dark current and flat-fielded, de-spiked, and calibrated, but not exposure-time corrected. The filtergrams were then processed with aia_prep.pro, which adjusts the images to a common plate scale so that they share the same centers and rotation angles. The AIA images are usually taken with fixed exposure times, which can cause saturation during the peak phase of the flare. To avoid this, the exposure times can be automatically reduced during flares. We used these special short-exposure frames in our analysis when they were available.

The Reuven Ramaty High Energy Solar Spectroscopic Imager (RHESSI; Lin et al. 2002) has observed solar X-ray and gamma-ray emission from $3 \mathrm{keV}$ to $17 \mathrm{MeV}$ since its launch in 2002. It provides simultaneous imaging and spectroscopy with high time- and energy resolution as diagnostic tools of heated flare plasma with temperatures in excess of about $10 \mathrm{MK}$ and nonthermal bremsstrahlung that is due to accelerated electrons.

We also used magnetograms from the Helioseismic and Magnetic Imager (HMI; Scherrer et al. 2012) for context purposes. HMI data were also processed with aia_prep.pro to place them on the same plate scale as the AIA images.

All data were precisely spatially co-aligned before further analysis. The coordinate system of AIA was used as reference. The position of the EIS slit relative to the AIA images was obtained using cross-correlation techniques. To verify the final alignment, space-time maps of selected EIS and AIA intensity channels were constructed using the derived slit positions and positions shifted for several pixels in all directions. These 2D maps were again cross-correlated to find the best match. This method allowed us to co-align EIS and AIA data with a precision of around $1 "$, and the position of EIS slit center was found to be at $(x, y)=\left(448.6^{\prime \prime},-46.4^{\prime \prime}\right)$. RHESSI data are generally well aligned with the AIA filtergrams within $1^{\prime \prime}-2^{\prime \prime}$.

As the plasma parameters evolve quickly during the impulsive flare phase, it is important to state observation times precisely (especially if data from several instruments were taken with very different exposures). Therefore we always use the midpoint of the particular exposure or integration time (given in UT), respectively, if we refer to individual exposures of EIS, AIA, HMI, and RHESSI.

\section{Results}

\subsection{Event overview}

The temporal evolution of the RHESSI 3-100 keV count rates and GOES 1-8 $\AA$ SXR flux are plotted in Fig. 1. The recorded GOES 3-s data show that the flare started at 14:19 UT. The gradual increase in the flux changed to impulsive slightly before 14:22 UT, and the flare peaked at 14:25 UT (classifying the event as an M1.6 flare). Afterward, the X-ray flux decreased gradually. A similar temporal evolution is also seen in the lowenergy RHESSI light curves (from 3 to $12 \mathrm{keV}$ ), which are dominated by thermal emission from the hot coronal flare plasma. In contrast, the high-energy RHESSI curves (25 to $100 \mathrm{keV}$ ) show a different behavior. A gradual increase is followed by a sharp peak (especially the 25-50 keV channel) at 14:23:38 UT. The RHESSI high-energy light curves result from nonthermal bremsstrahlung emission from flare-accelerated electrons and are therefore a proxy for the evolution of the energy deposition rate in the flare. This implies that the main energy deposition occurred roughly $1.5 \mathrm{~min}$ before the flare reached its soft X-ray (SXR) peak. This time delay may be attributed to the characteristic time of chromospheric evaporation flows, filling the flare loop (governed by the sound speed). From the RHESSI 4-10 keV images, derived over the flare HXR peak shown in Fig. 2, we estimate the half-length of the flare loop as about $10 \mathrm{Mm}$. The fastest evaporation flows derived from the EIS spectroscopy (cf. Sect. 3.3) have speeds of 80 to $150 \mathrm{~km} \mathrm{~s}^{-1}$. The resulting loop filling time, derived from these numbers, lies 


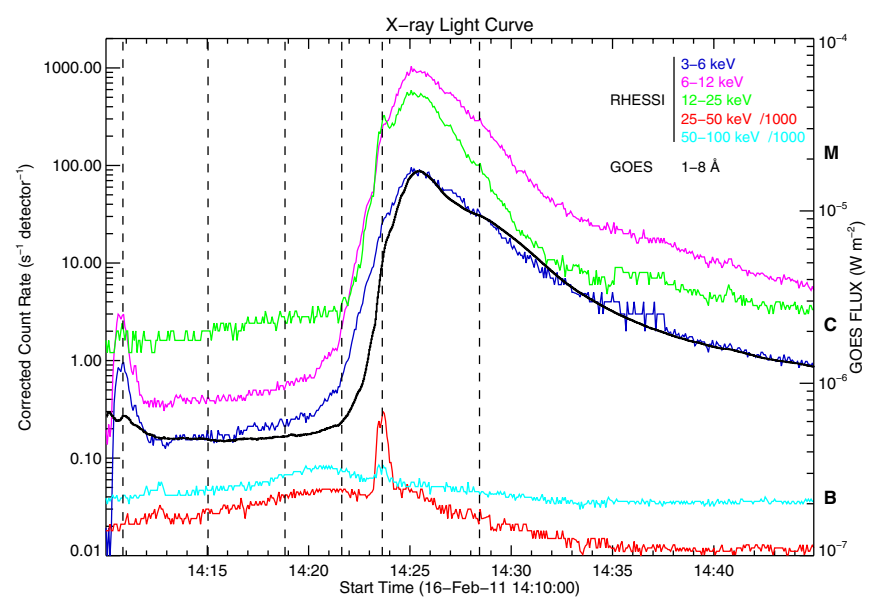

Fig. 1. RHESSI (3-100 keV, color-coded lines) and GOES (1-8 $\AA$, black line) light curves showing the temporal evolution of the X-ray flux during the M1.6 flare. The vertical dashed lines indicate the selected times for AIA images and RHESSI sources shown in Fig. 4.

in the range of 70 to $120 \mathrm{~s}$, which means that it is consistent with the observed delay between the peaks of the HXR and SXR curves.

Context images taken by the SDO/HMI (magnetograms and continuum intensity) and the SDO/AIA (filtergrams at different wavelengths and temperatures) instrument showing NOAA 11158 during the impulsive phase of the flare are shown in Fig. 2. The AIA filtergrams taken with short exposures (compared to standard observations) were used to visualize the impulsive phase of the flare. RHESSI CLEAN images integrated over $40 \mathrm{~s}$ starting at 14:23:08 UT (i.e., covering the HXR peak) are overplotted in Fig. 2. The flare was rather compact, showing signatures of two flaring footpoints (RHESSI 20-50 keV contours) that are connected by a loop top source (RHESSI 4-10 keV contours).

Figure 3 depicts the temporal evolution of the flare as observed in the AIA $94 \AA$, $171 \AA$, and $304 \AA$ filters. About 10 min before the flare, a newly formed loop-like structure appears in all these wavelength channels. The exact times of the first appearance of this feature are 14:09:57 UT for $304 \AA$, 14:10:13 UT for $171 \AA$, and 14:10:51 UT for $94 \AA$, and it is highlighted by an arrow in the second panel of the particular wavelength channels (Fig. 3; note that filtergrams taken slightly later than the time of the first appearance of the loop-like structure are used because they allow an easier identification of the discussed structure). After its first occurrence, the brightness of the loop-like structure increased slowly until $\sim 14: 22: 30$ UT (the nominal flare onset). The actual flare site is co-spatial with the western footpoint of the loop-like structure and is located very close to the EIS slit position. As the flare evolved, filament material was ejected in the form of two clouds (marked by arrows in the 5th and 6th panels of each wavelength channel in Fig. 3). The erupting filament material is traceable in the form of enhanced intensity in all analyzed channels and partly propagated along the EIS slit. Between 14:30 UT and 14:40 UT, the newly evolved system of the flare loops was partly covered by the EIS slit (best visible in the AIA $94 \AA$ A channel).

The early flare evolution, covering the pre-flare and impulsive phase, is visualized in Fig. 4, showing a sequence of AIA $94 \AA$ images together with co-temporal RHESSI 410 and $20-50 \mathrm{keV}$ sources in the form of contours. The
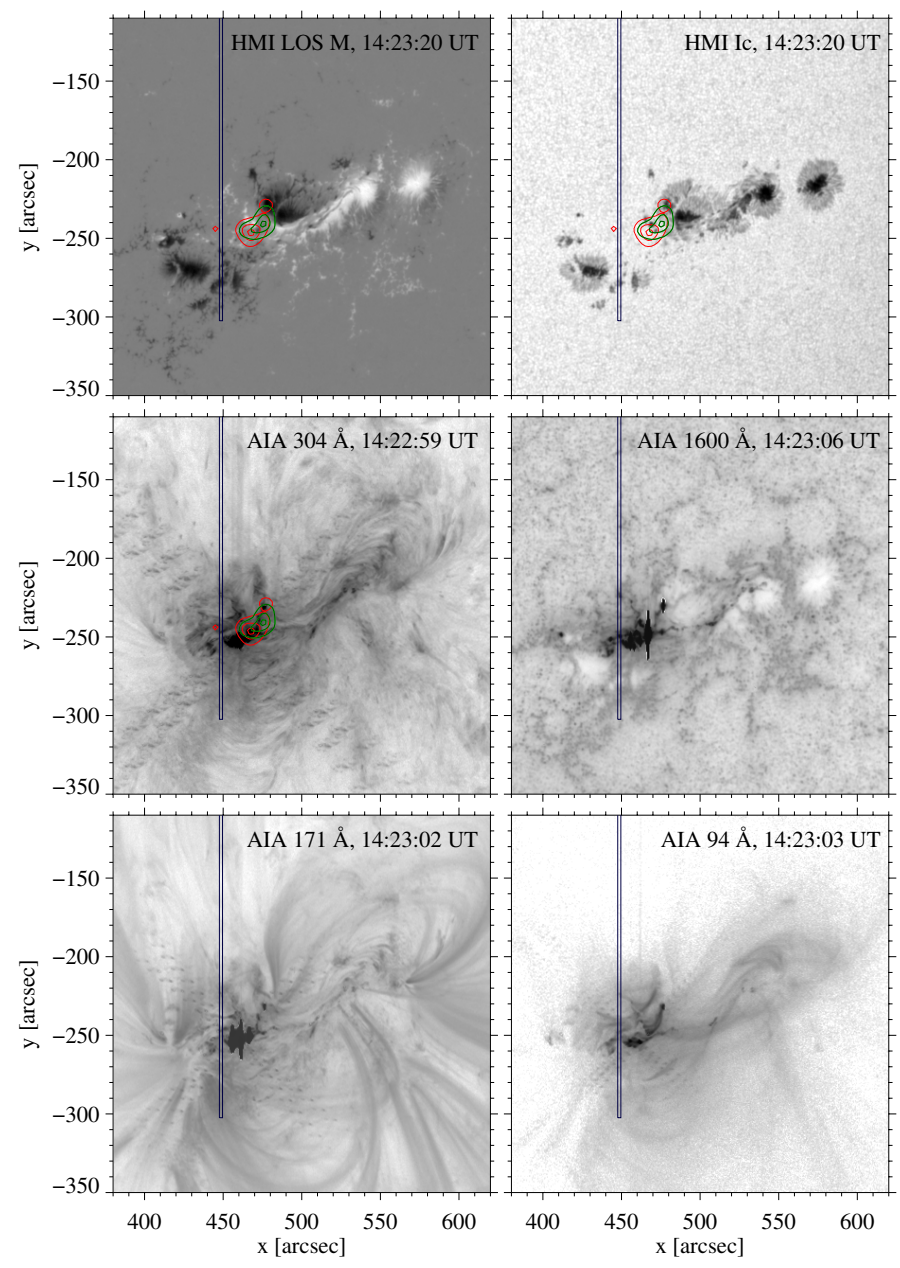

Fig. 2. Overview images showing NOAA 11158 during the main impulsive phase of the flare. Top left and right panels show the HMI line-ofsight magnetic field and continuum intensity, respectively. Middle and bottom panels are AIA filtergrams at different wavelengths (indicated in the top right corner of each panel). They are displayed on logarithmic scale and a reversed intensity scaling. The times listed in the upper right corner of each panel correspond to the midpoint of the particular HMI or AIA exposure time. The overplotted contours show RHESSI X-ray images reconstructed in the 4-10 keV (green) and 20-50 keV (red) energy bands, with an integration time of $40 \mathrm{~s}$, starting at 14:23:08 UT. The contour levels represent 10,50 , and $90 \%$ of the peak intensity. The rectangle outlines the lower part of the Hinode/EIS slit.

RHESSI images were reconstructed using the CLEAN algorithm (Hurford et al. 2002) with detectors 2-7. The brightenings in the RHESSI 4-10 keV SXR image mark the flare onset at locations slightly away from the later main flare site (Fig. 4a). However, the next panel (Fig. 4b) clearly shows a stable patch of 4-10 keV emission exactly at the main flare site. The RHESSI SXR source is situated very close to the position of the newly developed loop structure marked in Fig. 3, which is later observed in AIA to be the commencement site of the main flare (cf. Fig. 4d).

\subsection{EIS spectroscopy of the filament eruption}

As mentioned before, the EIS observations captured part of the filament eruption. All Fe XIII $202 \AA$ spectral line profiles in the corresponding parts of the EIS slit exhibit a twocomponent shape with the second Gaussian component to the spectral line profile shifted to shorter wavelengths. Examples 
P. Gömöry et al.: Chromospheric evaporation flows and density changes deduced from Hinode/EIS during an M1.6 flare
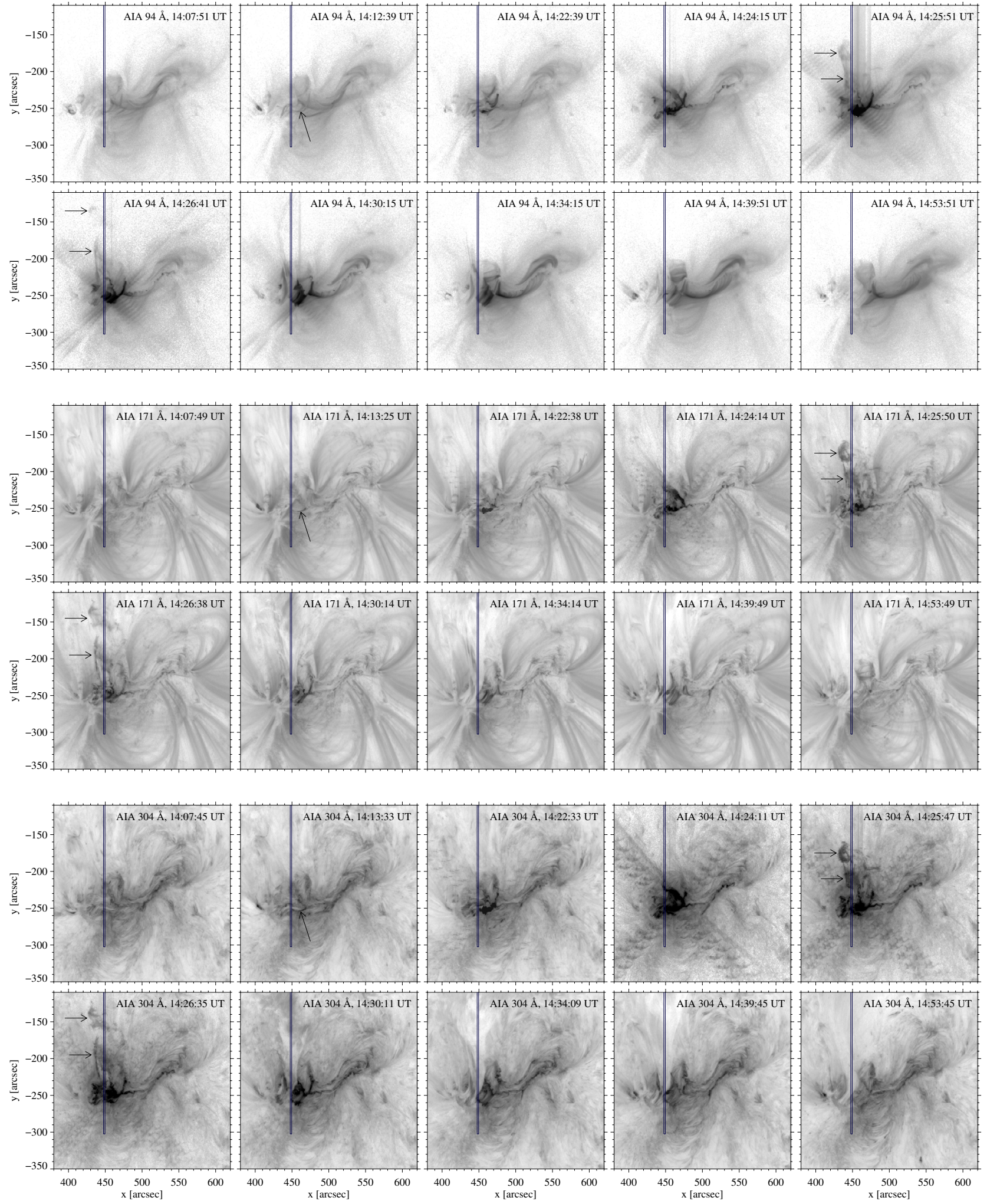

Fig. 3. Sequence of AIA $94 \AA$ (top two rows), $171 \AA$ (middle two rows), and $304 \AA$ (bottom two rows) filtergrams showing snapshots of the temporal evolution of the flare. The midpoint of the recording time of the particular images is presented in the upper right corner of each panel. The arrow in the second panel of each wavelength channel marks the appearance of a newly formed hot loop structure. The arrows in the 5th and 6th panel point to the erupting filament. The rectangles represent the position of the EIS slit. An animation of the displayed wavelength channels is shown in the attached movie.

of such spectral profiles are shown in Fig. 5. The detected blueshifts correspond to average Doppler velocities of around $-250 \mathrm{~km} \mathrm{~s}^{-1}$ and $-300 \mathrm{~km} \mathrm{~s}^{-1}$ for the first and second cloud of ejected filament material, respectively. These values can be used as a lower estimate of the real speed of the filament eruption. We note that the Fe XVI $262 \AA$ spectra were too noisy and weak in the filament to allow for a reliable analysis of this type. 


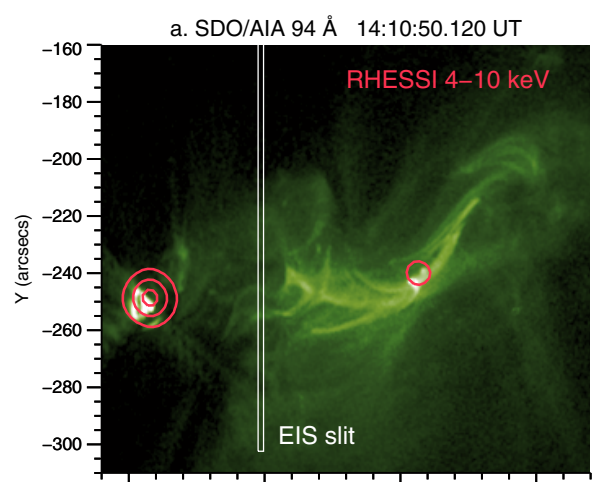

d. SDO/AIA $94 \AA$ A $14: 21: 38.120$ UT

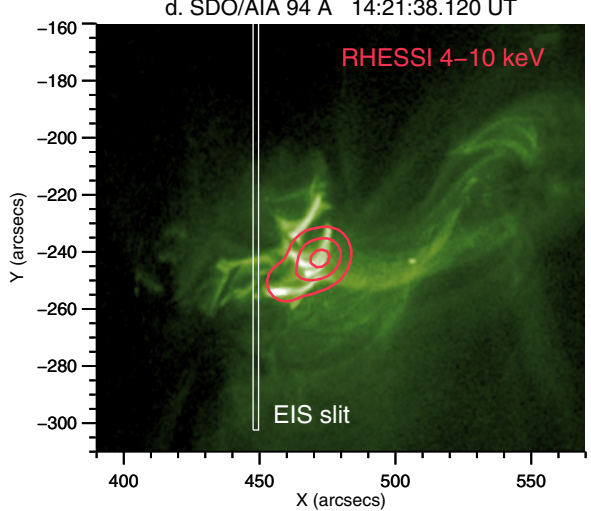

b. SDO/AIA $94 \AA \quad 14: 15: 02.120$ UT

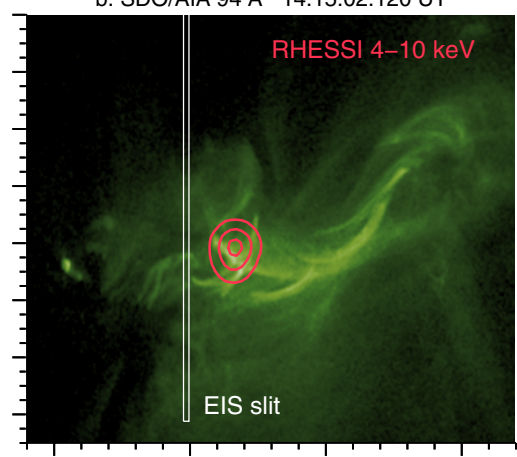

e. SDO/AIA $94 \AA \quad 14: 23: 38.120$ UT

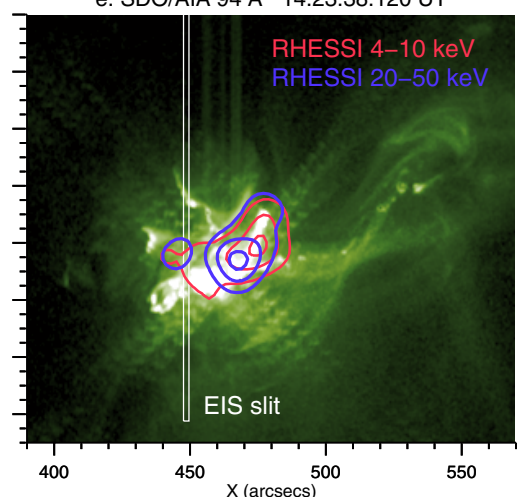

C. SDO/AIA $94 \AA \quad 14: 18: 50.120$ UT

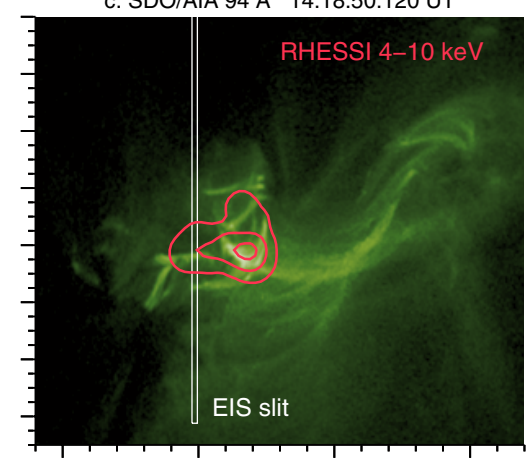

f. SDO/AIA $94 \AA \quad 14: 28: 26.120$ UT

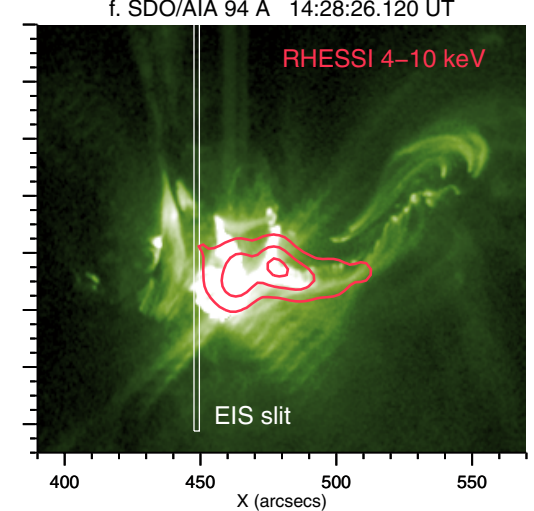

Fig. 4. SDO/AIA $94 \AA$ images at six different times, as indicated in the X-ray light curves plotted in Fig. 1. These images show the evolution of flare loops at a temperature around 6 MK. RHESSI X-ray sources are shown as contours in red (4-10 keV) and blue (20-50 keV). They show the locations of X-ray-emitting thermal plasma and nonthermal electrons. The contour levels are 5, 30, and $80 \%$ of the peak intensity. The white rectangle depicts the lower part of the Hinode/EIS slit.

\subsection{EIS spectroscopy of the flare}

Figure 6 gives an overview of the spectroscopic parameters (intensities and Doppler shifts), determined from the Fe XIII $202 \AA$ and Fe XVI $262 \AA$ spectral lines. The time-space maps show several structures such as the propagating EIT wave, the erupting filament material, and the flare site (cf. Veronig et al. 2011; Harra et al. 2011). In the following, we concentrate on a small fraction along the EIS slit (roughly between -260" and $\left.-250^{\prime \prime}\right)$. Figure 7 shows that this part of the EIS slit was cospatial with one of the flare kernels (cf. also the attached movie). RHESSI imaging also demonstrates that the eastern flare kernel (compare the $20-50 \mathrm{keV}$ contour) is partially located at the EIS slit (Fig. 4e).

Figure 8 shows the temporal variations of the intensities (top panels) and Doppler shifts (bottom panels) that were extracted from four consecutive locations along the EIS slit covering the flare kernel. The variations represent the pre-flare, main impulsive phase, and post-flare time sequence. The exact location of the selected EIS pixels is marked in Fig. 7.

The flare commencement in the selected pixels is clearly visible in Fig. 8 in the form of a sharp increase in the intensities of the two lines we study. The intensity of the cooler line (Fe XIII $202 \AA$ ) reaches its maximum at 14:23:34 UT, the hotter line (Fe XVI $262 \AA$ ) reaches it one exposure later (at 14:24:21 UT). The Doppler shifts of the two lines behave differently. The Fe XIII $202 \AA$ emission is subject to weak blueshifts during the pre-flare phase, which gradually increase from values close to $0 \mathrm{~km} \mathrm{~s}^{-1}$ at 14:14:12 UT to velocities of around $-10 \mathrm{~km} \mathrm{~s}^{-1}$, measured just before the peak of the spectral line intensity. We assume that this represents slowly expanding plasma that was heated before the impulsive phase of the flare. On the other hand, the Fe XVI $262 \AA$ line exhibited no obvious Doppler shifts, indicating that the local atmospheric plasma at this temperature was still at rest during the pre-flare phase. Then, at the moment of the main intensity peak, the Fe XIII $202 \AA$ line exhibits weak redshifts of $2-3 \mathrm{~km} \mathrm{~s}^{-1}$. These redshifts were only detected for a single exposure. Because the uncertainty in the Doppler shift calculation introduced by the HK method (i.e., wavelength drift compensation) corresponds to about $4.4 \mathrm{~km} \mathrm{~s}^{-1}$, however, the estimated redshifts are lower than the instrument resolution and are probably not reliable. Later on, again only blueshifts of about $-10 \mathrm{~km} \mathrm{~s}^{-1}$ and lower (approaching almost zero velocity at $\sim 14: 30 \mathrm{UT}$ ) were present. In contrast, during the flare impulsive phase, the Fe XVI $262 \AA$ velocities reveal only blueshifts. Their monotonic increase starts to appear roughly at 14:21 UT, and they reached maximum values of $\sim 55 \mathrm{~km} \mathrm{~s}^{-1}$ at the moment of the intensity peak. The velocities then started to decrease and revealed minimum values at the time interval 14:27-14:30 UT.

Figure 9 shows representative spectra of each flare phase (pre-flare, impulsive, and decay). Close inspection reveals that the Fe XVI $262 \AA$ spectral profiles exhibit a significant asymmetry during the impulsive phase. When approximated by two Gaussians, high-velocity components are derived, suggesting that a fraction of the plasma was moving upward with velocities of up to $80-150 \mathrm{~km} \mathrm{~s}^{-1}$. In contrast, only single-Gaussian spectral profiles were detected for the Fe XIII $202 \AA$ line during the whole flare evolution (they were just slightly broadened during 
P. Gömöry et al.: Chromospheric evaporation flows and density changes deduced from Hinode/EIS during an M1.6 flare
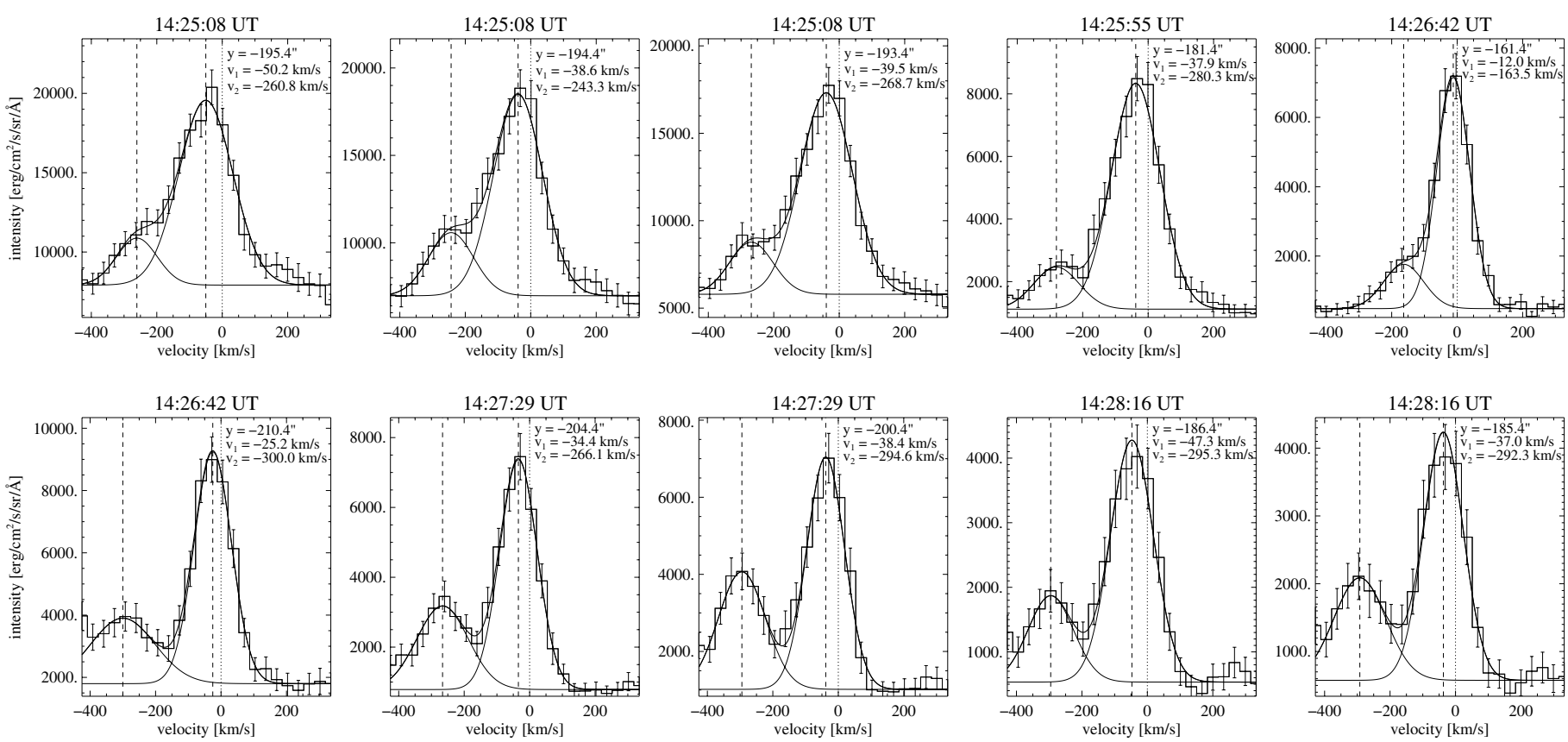

Fig. 5. Representative examples demonstrating the two-component character of the Fe XIII $202 \AA$ spectral profiles related to the erupting filament material (the top row represents the leading and the bottom row the following part of the ejected filament marked by the arrows in the 5th and 6th panel of Fig. 3). The histograms show the observed spectral profiles. The standard deviation of the line intensity is indicated by error bars. The individual spectral components and the resulting fits are plotted with solid lines. Vertical dotted lines show the position of the zero Doppler shifts, while the vertical dashed lines highlight the values of Doppler shifts determined for particular components of the fit. The positions along the slit together with the determined values of Doppler shifts are given in the upper right corner of each panel.

the impulsive phase). However, a significant intensity increase is visible in the very far blue wing of this line (Fig. 9, top row). If this spectral component were related to the flare, then it would represent upflows with extremely high Doppler shifts (more then $450 \mathrm{~km} \mathrm{~s}^{-1}$ ), which are sometimes observed during flares in spectral lines formed at temperatures higher than $10 \mathrm{MK}$, but not at temperatures sampled by Fe XIII spectral lines. However, this enhancement might also be the signature of another spectral line that becomes strong when the local atmosphere began to be heated. The CHIANTI spectral line list suggests that it may be a blend of Fe XI and Fe XII around $201.74 \AA$. This blend is at just the right wavelength to imply a blueshifted Fe XIII component with a velocity corresponding to about $450 \mathrm{~km} \mathrm{~s}^{-1}$. This explanation is also supported by the fact that the feature is visible well before the impulsive flare phase (e.g., at 14:14:12 UT, as is shown in Fig. 9).

The impulsive phase of the flare evolution is accompanied by a rapid enhancement in the electron densities (Fig. 10) computed from the intensity ratio of the Fe XIII spectral lines observed at 196 and $202 \AA$. This line pair was selected for the density analysis instead of the available Fe XIII 203/202 A line pair because it is more sensitive to the higher densities expected during the flare. The calculated electron densities rise from a preflare level of $\log \left(n_{\mathrm{e}}\right)=\sim 9.7$ (i.e., $5.01 \times 10^{9} \mathrm{~cm}^{-3}$ ) to values of up to $\log \left(n_{\mathrm{e}}\right)=\sim 10.5$ (i.e. $3.16 \times 10^{10} \mathrm{~cm}^{-3}$ ) within less than two minutes during the impulsive phase.

During the decline phase, the Doppler shifts of both Fe XIII $202 \AA$ and Fe XVI $262 \AA$ lines exhibit secondary peaks of blueshifts with maximum values of $\sim 15 \mathrm{~km} \mathrm{~s}^{-1}$ in the time interval between 14:31 UT and 14:36 UT (Fig. 8). Spectral profiles typical for this phase are displayed in Fig. 9 (last column). They confirm that the lines do not exhibit multi-component shapes at this time. The detected upflows correspond to a significant intensity increase in the Fe XVI $262 \AA$ line. Gentle intensity growth is also visible in the Fe XIII $202 \AA$ line. Moreover, the electron densities (Fig. 10) determined from the pair of Fe XIII lines exhibit a significant peak with $\log \left(n_{\mathrm{e}}\right)=\sim 10.07$ (i.e. $\left.1.17 \times 10^{10} \mathrm{~cm}^{-3}\right)$. We note that the intensity peaks of both lines and the density peak are delayed, but they persist much longer than the corresponding peaks visible in the Doppler shifts (compare Figs. 8 and 10). The increase of the electron densities and intensities together with the detected upflows can be explained by a process that fills the loops with expanding hot material that is due to chromospheric evaporation. This interpretation is also supported by the fact that a new system of loops was observed at the EIS slit location at times that correspond to the discussed secondary density enhancement (see also Fig. 3).

\subsection{RHESSI X-ray spectroscopy of the flare}

Figure 11 displays three RHESSI X-ray spectra together with the fitting results to show the evolution of the hot plasma and accelerated electrons during the rising phase, around the HXR peak time, and during the decay phase. These spectra were derived from detector 4 with $20 \mathrm{~s}$ integration time and were fit with an isothermal component and an nonthermal thick-target model (Holman et al. 2003).

A second isothermal component was added to improve the fitting result whenever necessary (Fig. 11c). Following Feng et al. (2013) and Emslie et al. (2012), we estimated the nonthermal energy flux based on the fitting result around the HXR peak, which gives a value of $7.71 \times 10^{27} \mathrm{erg} \mathrm{s}^{-1}$. Taking the area enclosed by the $50 \%$ contour in the $20-50 \mathrm{keV}$ image (Fig. $4 \mathrm{e}$ ) as the cross section of the flaring loops, we estimated a nonthermal energy flux density of $1.34 \times 10^{10} \mathrm{erg} \mathrm{s}^{-1} \mathrm{~cm}^{-2}$, which is close to the threshold of $10^{10} \mathrm{erg} \mathrm{s}^{-1} \mathrm{~cm}^{-2}$ between explosive 

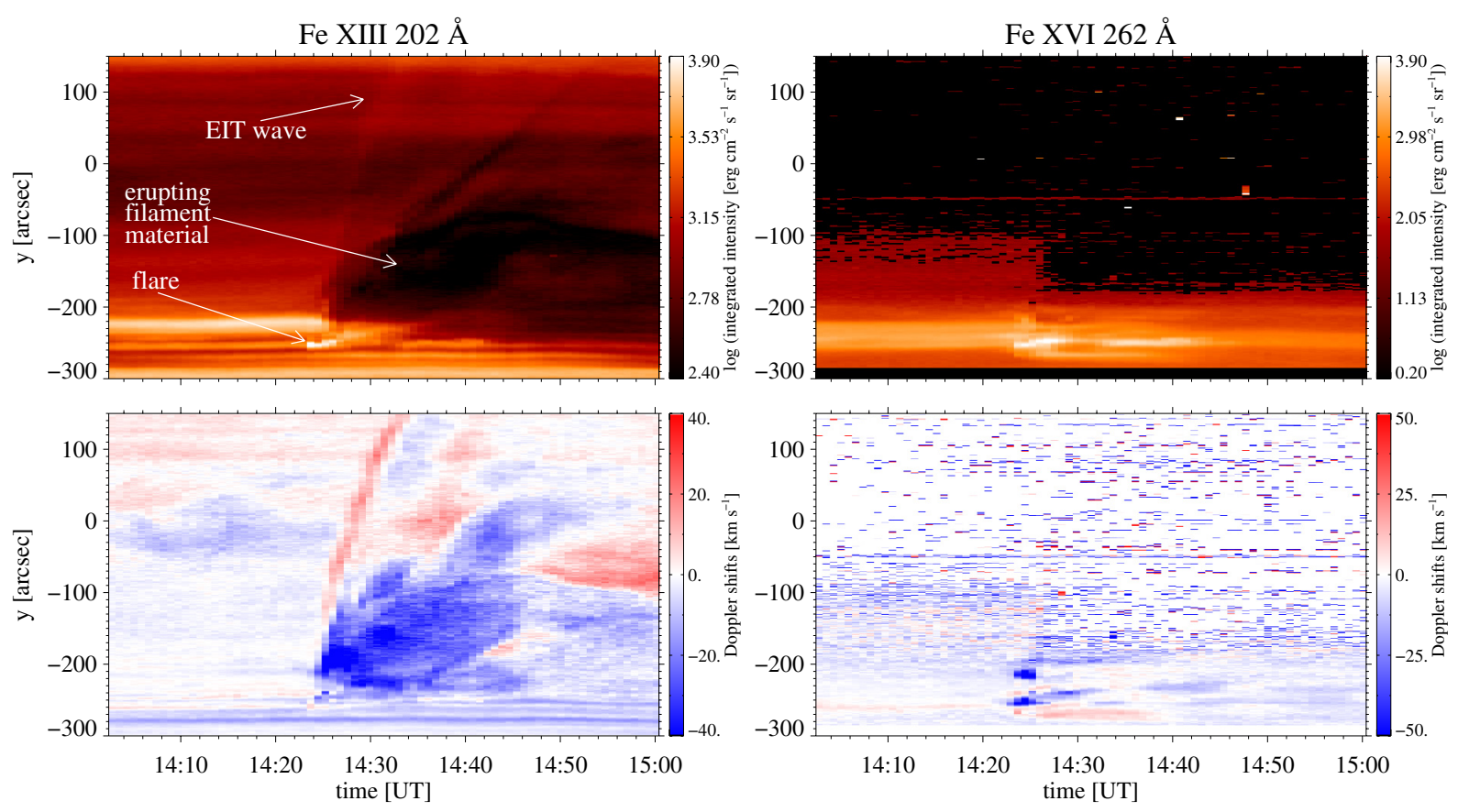

Fig. 6. Time-space maps of the Fe XIII $202 \AA$ and Fe XVI $262 \AA$ intensities (top panels) and Doppler shifts (bottom panels). The temporal evolution of the observed flare is visible in the region between $-260^{\prime \prime}$ and $-250^{\prime \prime}$ along the slit. Observed features (EIT wave, erupting filament, and flare) are marked by arrows in the Fe XIII $202 \AA$ intensity map.

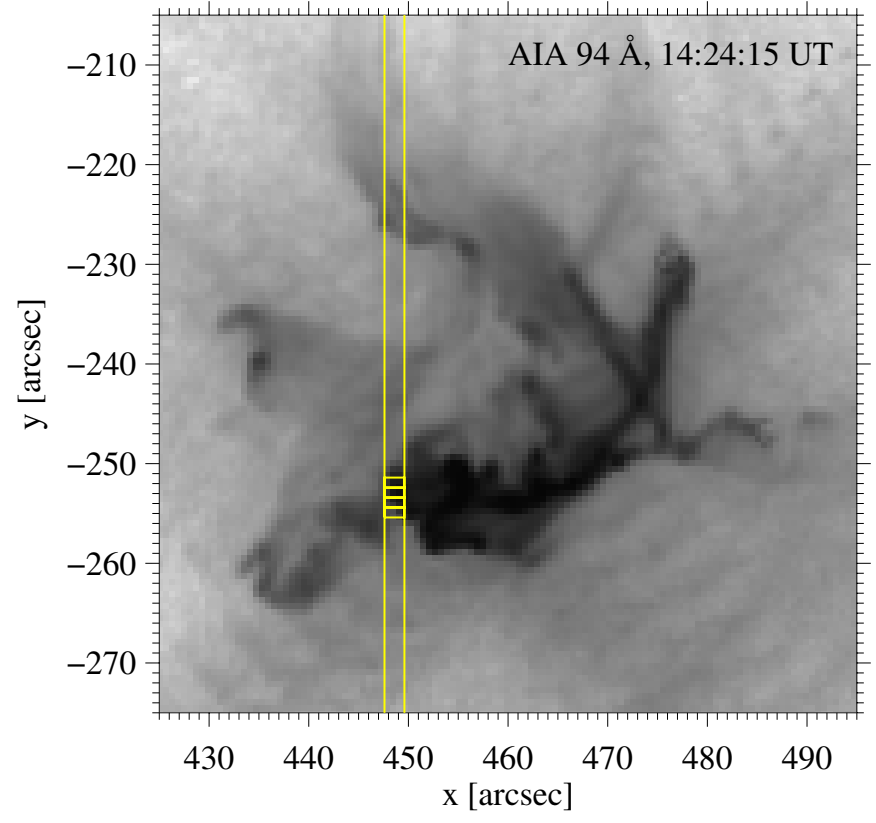

Fig. 7. Position of the EIS slit (yellow vertical lines) superimposed on an AIA $94 \AA$ image, taken during the impulsive phase of the flare at 14:24:15 UT. The yellow rectangles highlight the EIS pixels we used to study the temporal evolution of the intensities and Doppler shifts shown in Fig. 8.

and gentle evaporation derived from the hydrodynamic simulations by Fisher et al. (1985).

\section{Discussion}

The spectroscopic observations of the M1.6 flare under study revealed that the intensity maxima of the two analyzed spectral lines with different formation temperatures were not reached simultaneously (the intensity maximum of the hotter line follows the peak of the colder line by one exposure, i.e., by about $50 \mathrm{~s}$ ). However, both these times correspond well with the HXR peak time at 14:23:38 UT (Fe XIII $202 \AA$ intensity peaks at 14:23:34 UT and Fe XVI $262 \AA$ intensity at 14:24:21 UT). This suggests that the part of the EIS slit covering the flare ribbon (see Fig. 7) is located close to the site of the strongest energy deposition.

We detected strong upflows of up to $\sim 55 \mathrm{~km} \mathrm{~s}^{-1}$ in the hotter line (Fe XVI $262 \AA$ ) and no significant Doppler shifts (estimated downflows of $2-3 \mathrm{~km} \mathrm{~s}^{-1}$ are below the resolution of the spectrograph and thus are not very reliable) in the cooler line (Fe XIII $202 \AA$ ) during the impulsive phase of the flare. Moreover, a high-speed component was discovered in the Fe XVI $262 \AA$ spectra, suggesting that the flaring material expanded with velocities of up to $80-150 \mathrm{~km} \mathrm{~s}^{-1}$. In contrast, only single-component spectra were detected in the Fe XIII $202 \AA$ line during the impulsive and the decay phase of the flare evolution.

A dependency of the Doppler velocity directions on the formation temperature of spectral lines has been observed during the impulsive phases of flares. Kamio et al. (2005) reported that they did not detect any significant changes in the Doppler shifts of the MgIX spectral line. Therefore, they suggested that the observed plasma at $\log T=6.0$ was close to the intermediate temperature between the upflowing hot plasma $(\log T=7.0)$ and the downflowing chromospheric and transition region plasma ( $\log T=4.0-5.0)$. Milligan et al. (2006a) found strong blueshifts of up to $\sim 250 \mathrm{~km} \mathrm{~s}^{-1}$ co-spatial with flare ribbons using the Fe XIX spectral line (forming at $\log T=6.9$ ) and weak redshifts for the He I and OV spectral lines (sensitive to chromospheric and transition region temperatures, respectively). This is again suggestive of the temperature regime where Doppler velocities change from downflows to upflows. This was also confirmed by the first study of the impulsive phase of a flare observed with 
P. Gömöry et al.: Chromospheric evaporation flows and density changes deduced from Hinode/EIS during an M1.6 flare

Fe XIII $202 \AA$

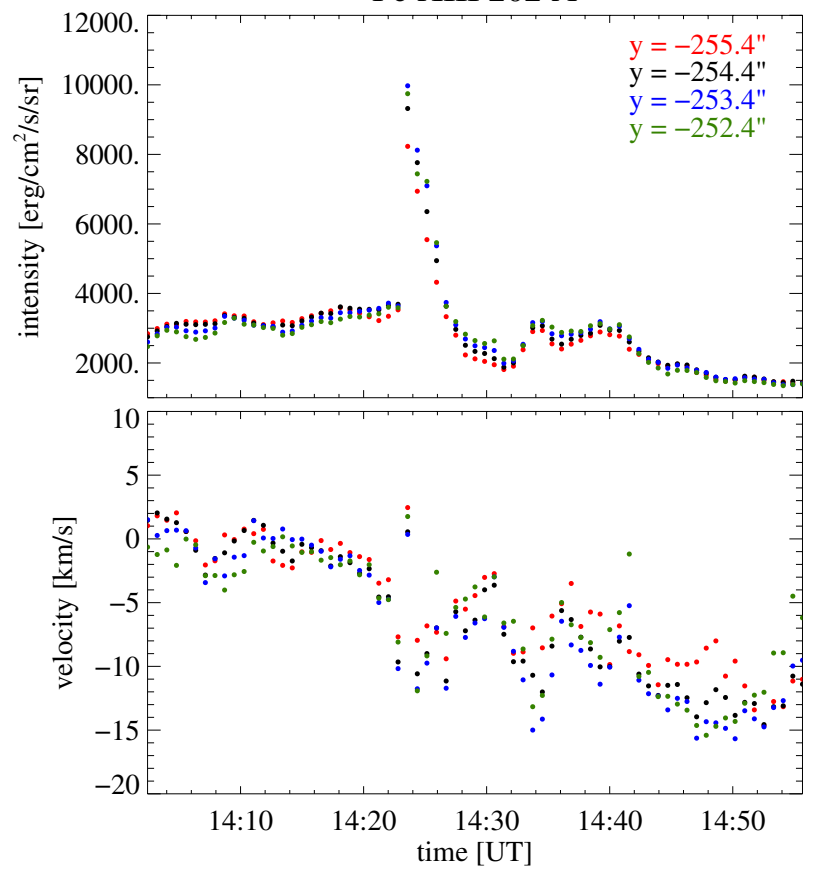

Fe XVI $262 \AA$

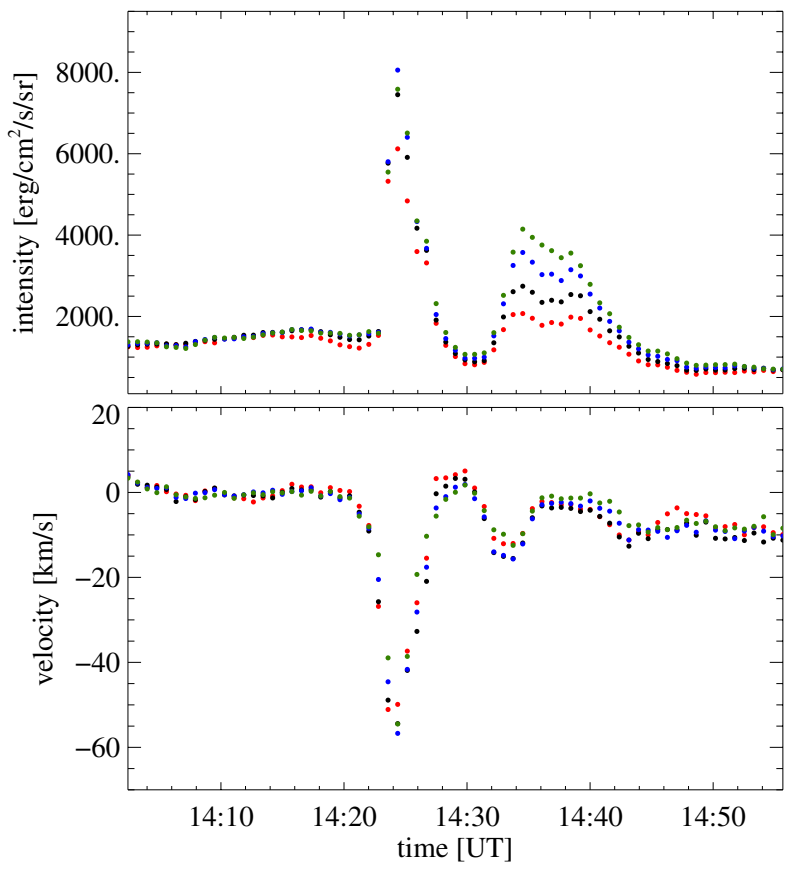

Fig. 8. Temporal evolution of the Fe XIII $202 \AA$ and Fe XVI $262 \AA$ intensities (top panels) and Doppler shifts (bottom panels), extracted from four consecutive pixels along the EIS slit that cover the flaring region. The pixels are indicated by yellow rectangles in Fig. 7.

the EIS spectrometer. Milligan \& Dennis (2009) observed footpoints of a $\mathrm{C} 1.1$ flare and found that the Doppler shifts revealed a change from redshifts to blueshifts at around $\log T=6.3$. In particular, they showed that the emission from spectral lines formed in the temperature range $\log T=6.3-7.2$ exhibited clear blueshifts that scaled with temperature and reached velocities of $>250 \mathrm{~km} \mathrm{~s}^{-1}$ for the hottest spectral line Fe XXIV. On the other hand, the emission formed at temperatures $\log T=4.7-6.2 \mathrm{was}$ found to be redshifted. In contrast, a detailed study of an M1.1 flare performed by Young et al. (2013) suggested that the change from redshift to blueshift appears between the Fe X and Fe XII spectral lines, that is, at a temperature of about $\log T=6.1$ (see Fig. 7 in their work). The lower temperature of the transition from redshifts to blueshifts was also reported by Graham et al. (2011). They observed small downflows at temperatures below Fe XIII $(\log T=6.2)$ and upflows of up to $\sim 140 \mathrm{~km} \mathrm{~s}^{-1}$ at higher temperatures. Our results indicate that the intermediate temperature separating hot upflowing and cooler downflowing material is approximately at $\log T=6.2$ because we did not detect any significant flows in the Fe XIII spectral line.

The observed change of the Doppler velocity direction during the impulsive phase of the flare is interpreted within the model of explosive chromospheric evaporation. In this scenario, hotter plasma rises toward the corona and cooler plasma falls toward the photosphere to preserve momentum balance.

Our analysis of the RHESSI spectra and images suggests that the energy flux deposited by the beam of accelerated electrons to the lower atmospheric layers was $1.34 \times 10^{10} \mathrm{erg} \mathrm{s}^{-1} \mathrm{~cm}^{-2}$ during the flare HXR peak. This value is very close to the theoretical threshold of $10^{10} \mathrm{erg} \mathrm{s}^{-1} \mathrm{~cm}^{-2}$ between gentle and explosive evaporation determined by Fisher et al. (1985). It is therefore somewhat puzzling that we found clear signatures of explosive evaporation under these conditions.

However, the results of Fisher et al. (1985) were derived under several assumptions. A critical one is the usage of a fixed low-energy cut-off $(20 \mathrm{keV})$ in their simulation. This assumes an abundance of high-energy electrons in the beam, but no particles with energies below $20 \mathrm{keV}$. In a recent study, Reep et al. (2015) performed detailed hydrodynamical simulations to examine the atmospheric response to heating by different isoenergetic beams of accelerated electrons. In particular, they analyzed the role of electron energy and stopping depths in the two regimes of chromospheric evaporation. They found that the threshold between explosive and gentle evaporation found by Fisher et al. (1985) depends quadratically on the electron energy and linearly on the beam flux. The threshold of $\sim 10^{10} \mathrm{erg} \mathrm{s}^{-1} \mathrm{~cm}^{-2}$ was confirmed for beams formed by electrons with energy $20 \mathrm{keV}$. But lower energy fluxes are sufficient to drive explosive evaporation if electrons with energies below $20 \mathrm{keV}$ are considered. We recall that in the flare under study, the fits to the RHESSI spectra indicate a low-energy cut-off of $16 \mathrm{keV}$ (cf. Fig. 11b), which is an upper limit, and the real cut-off might be even lower.

Moreover, Reep et al. (2015) pointed out that lower energy electrons are more efficient in heating flaring loops and driving dense plasma into the corona. For example, if electron beams with a constant energy flux density of $10^{10} \mathrm{erg} \mathrm{s}^{-1} \mathrm{~cm}^{-2}$ were considered, then those simulations using a high-energy electron beam showed only small electron density enhancements during the flare. In contrast, significant density increase only appears during a flare for beams with electron energies of $15 \mathrm{keV}$ and below. This agrees with our result, which shows a clear density enhancement co-temporal with the HXR peak, that is, for the time when a low-energy cut-off of $\lesssim 16 \mathrm{keV}$ was estimated from the RHESSI spectra.

The higher efficiency of the low-energy electrons in triggering explosive evaporation was explained in the following way by Reep et al. (2015). Electrons of lower energy are effectively stopped higher up in the atmosphere and deposit their energy in the low-density plasma which has less inertia and has a lower heat capacity. Consequently, the plasma pressure starts to rise and explosive evaporation sets in. On the other hand, the stopping depth of high-energy electrons can extend deep into the 

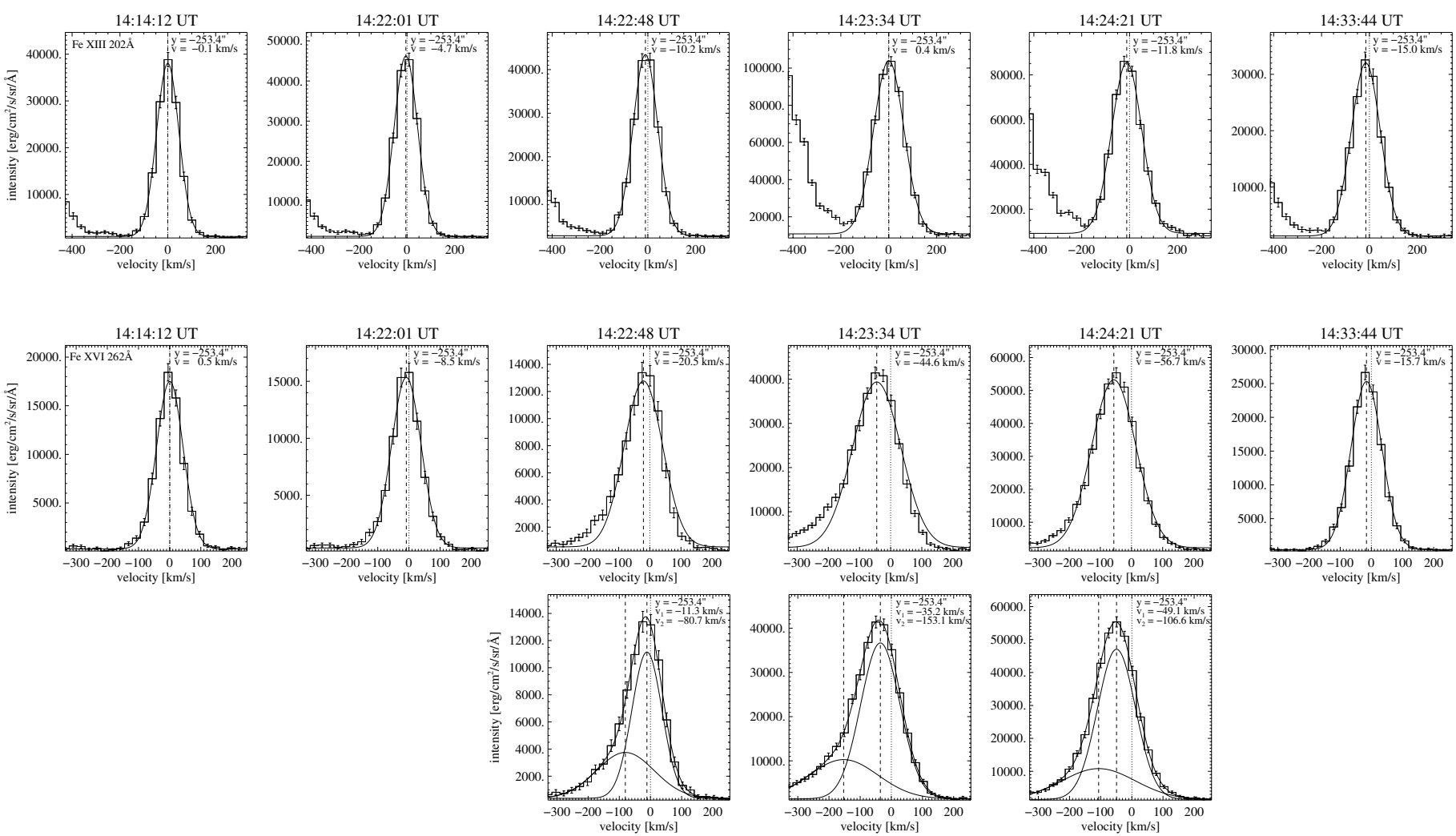

Fig. 9. Representative examples of Fe XIII $202 \AA$ (top row) and Fe XVI $262 \AA$ (middle and bottom rows) spectral line profiles and their fits for particular phases of the flare evolution, i.e., early pre-flare period (first column), impulsive phase (second to fifth column), and decline phase (last column). The top and middle rows show the profiles fitted by a single-Gaussian function. The bottom row displays results obtained from two-component fitting of the asymmetric Fe XVI $262 \AA$ spectral profiles detected during the impulsive phase of the flare. The histograms show the observed spectral profiles. The error bars mark the standard deviation for each intensity value. Solid lines represent final fits and individual spectral components (bottom row). Vertical dotted lines indicate the position of zero Doppler shifts, while the vertical dashed lines highlight the values of Doppler shifts determined for particular components of the fit. The positions along the slit together with the determined values of Doppler shifts are inserted in the upper right corner of each panel.

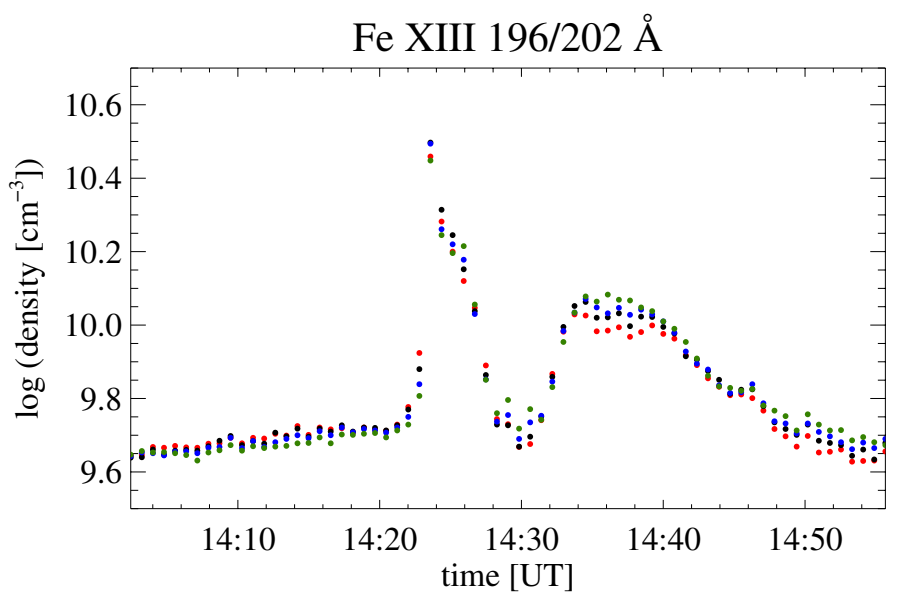

Fig. 10. Temporal variations of the electron density determined using the Fe XIII 196/202 Å line pair. The displayed values were selected from the same spatial pixels as intensities and Doppler shifts shown in Fig. 8.

solar atmosphere where the ambient density is much higher. In addition, this part of the atmosphere has a larger heat capacity and stronger radiative losses. Thus, the deposited energy is substantially lower than the local thermal energy. This explains why we observed clear evidence of explosive evaporation although the estimated energy flux density was close to $10^{10} \mathrm{erg} \mathrm{s}^{-1} \mathrm{~cm}^{-2}$.
We found significant enhancement of the electron densities during the impulsive flare phase, increasing from the pre-flare value of $\sim 5.01_{-0.137}^{+0.140} \times 10^{9} \mathrm{~cm}^{-3}$ to $\sim 3.16_{-0.135}^{+0.141} \times 10^{10} \mathrm{~cm}^{-3}$ within less than two minutes. This maximum occurs at the same time as the Fe XIII $202 \AA$ velocities exhibit weak redshifts and the intensities peak sharply. However, it appears about 50 s (i.e., one exposure) earlier than the Fe XVI $262 \AA$ intensities and Doppler shifts reached their maxima.

The densities were determined using the Fe XIII 196/202 $\AA$ line pair and therefore correspond to temperatures around $1.58 \mathrm{MK}(\log T=6.2)$. Similar values of electron densities were determined by Graham et al. (2011), who measured density enhancements of up to a few $10^{10} \mathrm{~cm}^{-3}$ in the flare footpoints at temperatures of $\sim 1.5-2 \mathrm{MK}$. Watanabe et al. (2010) also observed an increase of the electron densities of up to $3 \times 10^{10} \mathrm{~cm}^{-3}$ at evaporation sites. Young et al. (2013) used the Fe XIV 264.79/274.20 (formed at 2 MK) spectral line ratio and determined electron densities of $\sim 3.4 \times 10^{10} \mathrm{~cm}^{-3}$ co-spatial with a flare kernel. We note that while the findings of the latter authors were based on raster observations, we used sit-and-stare measurements for our study. The first EIS measurement of a flare using the sit-and-stare observing mode was performed by Brosius (2013a). He observed a C1 flare and determined the electron densities using a pair of Fe XIV spectral lines. The analysis yielded an average pre-flare value of $3.75 \times 10^{9} \mathrm{~cm}^{-3}$ and an average flare value of $4.94 \times 10^{9} \mathrm{~cm}^{-3}$. The reported lower density increase is very probably related to the fact that 

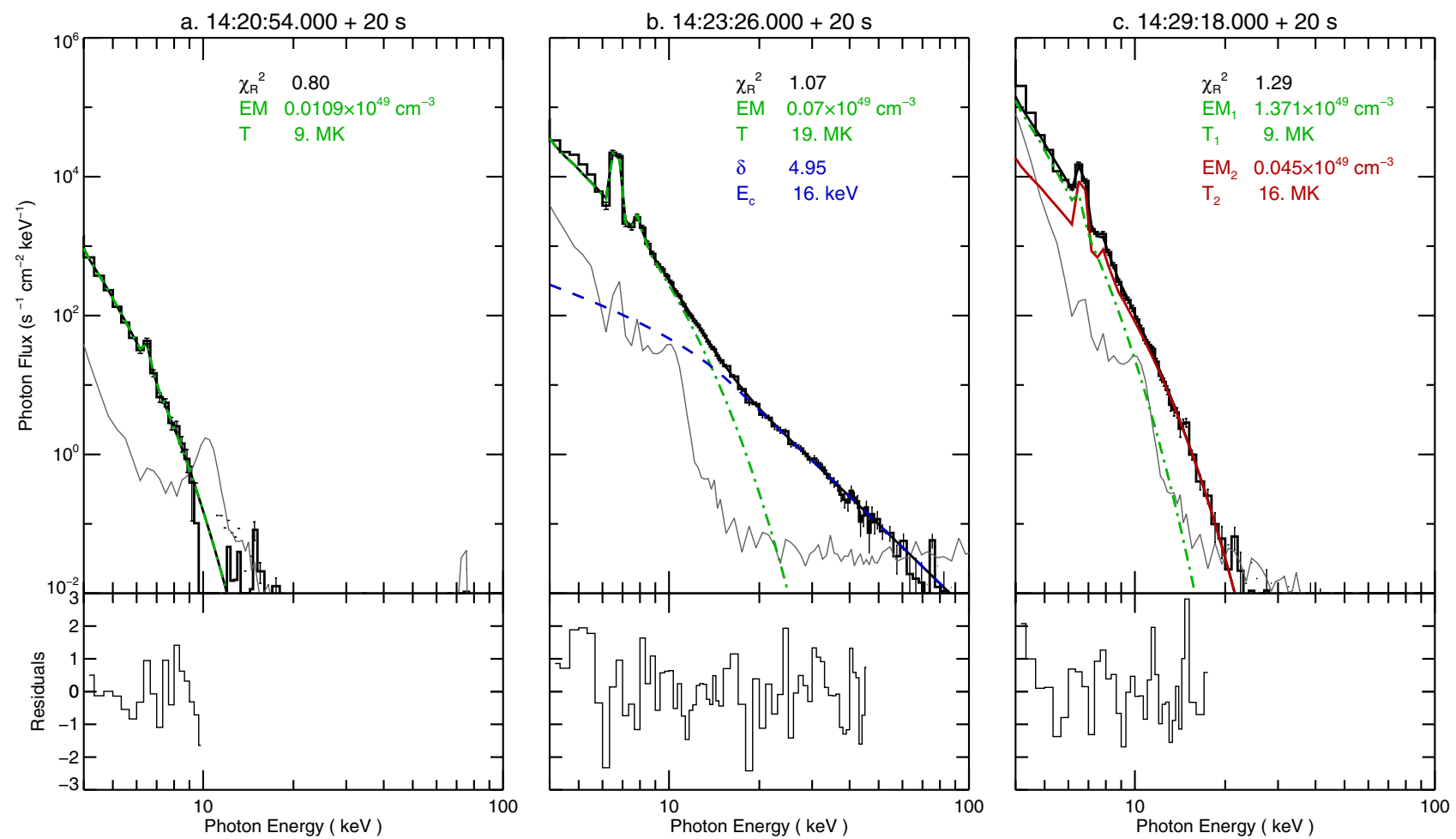

Fig. 11. Panels a) to c), top: reconstructed X-ray spectra and fitting results. The three examples shown here were selected at the rising phase, the HXR peak, and the decay phase. These spectra were derived from detector 4 with $20 \mathrm{~s}$ integration time (histogram) and fitted with an isothermal component (green, dot-dashed line) and a nonthermal thick-target model (blue, dashed line). A second isothermal component (red, solid line) was added when necessary for a better fit. The gray curves represent the background. The obtained parameters, such as reduced chi-square, plasma temperature $T$, emission measure $E M$, power-law distribution index $\delta$ of the nonthermal electrons, and low-energy cutoff $E_{c}$, are listed in the top right corner of each panel. Panels a) to c), bottom: residuals from the fitting.

the flare under study was a GOES C-class event. On the other hand, analysis of another C1 flare presented by Brosius (2013b) showed that the electron densities derived again from a pair of Fe XIV spectral lines increased by an order of magnitude from the pre-flare value of $3.43 \times 10^{9} \mathrm{~cm}^{-3}$ to its maximum impulsive phase value of $3.04 \times 10^{10} \mathrm{~cm}^{-3}$ in two minutes. This is in quantitative agreement with our findings, although we analyzed a stronger (GOES M1.6) flare. Therefore it suggests that the flare-induced changes of the electron densities are not strongly dependent on the flare energy.

The observed enhancement of the electron density during the main phase of the flare can be explained in the following way. Thermal and nonthermal energy released during the impulsive phase of the flare is deposited at deeper layers of the solar atmosphere, which consequently heat up. Thus, emission at coronal temperatures is released from much denser regions, explaining the observed increase. An alternative explanation is that the high-energy electrons that produce the observed HXR emission during the flare impulsive phase can easily penetrate the partially neutral atmosphere below where they are effectively stopped by collisions. This process can contribute to the ionization of the surrounding plasma and also lead to local heating and can be partly responsible for the measured enhancements of electron densities.

After the impulsive flare phase, we found peaks of blueshifts reaching $\sim 15 \mathrm{~km} \mathrm{~s}^{-1}$ for the two spectral lines under study at the time interval between 14:31 UT and 14:36 UT. These were followed by intensity enhancements in both lines that appear two exposures (i.e., $\sim 100 \mathrm{~s}$ ) later and by a significant increase of the electron densities, with the same time delay. We found that the footpoints of the flare loops were located co-spatial with the EIS slit at that particular moment. Therefore these observational facts provide evidence that the heated flaring plasma was upflowing and thus filling newly created magnetic loops that subsequently emit the observed radiation.

\section{Conclusions}

We presented EIS sit-and-stare spectroscopy of an M1.6 flare together with RHESSI X-ray observations. During the impulsive phase, we detected different behavior of Doppler shifts in the spectral lines under study, which are formed at slightly different temperatures. In particular, we found insignificant downflows in the Fe XIII $202.044 \AA(\log T=6.2)$ line and strong upflows in the Fe XVI $262.980 \AA(\log T=6.4)$ line. These flows were related to strong intensity increases observed in both lines and also to a significant increase of the electron densities measured at $1.56 \mathrm{MK}$ from about $5.01 \times 10^{9} \mathrm{~cm}^{-3}$ to $3.16 \times 10^{10} \mathrm{~cm}^{-3}$. In summary, the spectroscopic analysis suggests that explosive chromospheric evaporation took place during the flare peak. However, RHESSI X-ray spectroscopy interpreted with a collisional thick target model provided an estimate of the nonthermal energy flux density on the order of $\sim 10^{10} \mathrm{erg} \mathrm{s}^{-1} \mathrm{~cm}^{-2}$, meaning that the energy deposited in the lower solar atmosphere was on the level considered as the threshold between gentle and explosive evaporation in hydrodynamic simulations (e.g., Fisher et al. 1985). These findings provide evidence that the response of the flaring atmosphere strongly depends on the properties of the heating electron beams, which agrees with the predictions derived from 
recent numerical simulations (Reep et al. 2015). During the decline phase, we detected a secondary peak of intensities and electron densities preceded by upflows in both observed lines. These were interpreted as signatures of flare loops filled by the hot material that expands as a result of chromospheric evaporation.

Acknowledgements. This work was supported by the project of the Österreichischer Austauschdienst (OeAD) and the Slovak Research and Development Agency (SRDA) under grant Nos. SK 16/2013 and SK-AT-000312. P.G. acknowledges the support from grant VEGA 2/0004/16 of the Science Grant Agency and from the project of the Slovak Research and Development Agency under the Contract No. APVV-0816-11. A.M.V. and Y.S. gratefully acknowledge support from the Austrian Science Fund (FWF) P27292. Y.S. also acknowledges the Thousand Young Talents Plan, a sub-program of the "Recruitment Program of Global Experts" (1000 Talent Plan), and 11233008 from NNSFC. J.K.T. acknowledges support from Austrian Science Fund (FWF) P25383-N27. This article was created by the realization of the project ITMS No. 26220120009, based on the supporting operational Research and development program financed from the European Regional Development Fund. The authors thank an anonymous referee for constructive comments and valuable suggestions that improved this paper. Hinode is a Japanese mission developed and launched by ISAS/JAXA, with NAOJ as domestic partner and NASA and STFC (UK) as international partners. It is operated by these agencies in cooperation with ESA and NSC (Norway). SDO is a mission for NASA's Living With a Star (LWS) Program. CHIANTI is a collaborative project involving the NRL (USA), the Universities of Florence (Italy) and Cambridge (UK), and George Mason University (USA). RHESSI is a NASA small explorer mission. This research has made use of NASA's Astrophysics Data System.

\section{References}

Allred, J. C., Hawley, S. L., Abbett, W. P., \& Carlsson, M. 2005, ApJ, 630, 573 Antonucci, E., Gabriel, A. H., Acton, L. W., et al. 1982, Sol. Phys., 78, 107

Antonucci, E., Alexander, D., Culhane, J. L., et al. 1999, in The many faces of the sun: a summary of the results from NASA's Solar Maximum Mission, eds. K. T. Strong, J. L. R. Saba, B. M. Haisch, \& J. T. Schmelz, 331

Aulanier, G., Pariat, E., Démoulin, P., \& DeVore, C. R. 2006, Sol. Phys., 238, 347

Benz, A. O. 2008, Liv. Rev. Sol. Phys., 5, 1

Berkebile-Stoiser, S., Gömöry, P., Veronig, A. M., Rybák, J., \& Sütterlin, P. 2009, A\&A, 505, 811

Brosius, J. W. 2001, ApJ, 555, 435

Brosius, J. W. 2013a, ApJ, 762, 133

Brosius, J. W. 2013b, ApJ, 777, 135

Brosius, J. W., \& Daw, A. N. 2015, ApJ, 810, 45

Brosius, J. W., \& Phillips, K. J. H. 2004, ApJ, 613, 580

Brown, J. C. 1971, Sol. Phys., 18, 489

Carmichael, H. 1964, NASA SP, 50, 451

Culhane, J. L., Harra, L. K., James, A. M., et al. 2007, Sol. Phys., 243, 19

Czaykowska, A., De Pontieu, B., Alexander, D., \& Rank, G. 1999, ApJ, 521, L75

Del Zanna, G., Berlicki, A., Schmieder, B., \& Mason, H. E. 2006, Sol. Phys., 234, 95

Del Zanna, G., Mitra-Kraev, U., Bradshaw, S. J., Mason, H. E., \& Asai, A. 2011, A\&A, 526, A1

Demoulin, P., van Driel-Gesztelyi, L., Schmieder, B., et al. 1993, A\&A, 271, 292

Dere, K. P., Landi, E., Mason, H. E., Monsignori Fossi, B. C., \& Young, P. R. 1997, A\&AS, 125, 149

Doschek, G. A., \& Warren, H. P. 2005, ApJ, 629, 1150

Doschek, G. A., Feldman, U., Kreplin, R. W., \& Cohen, L. 1980, ApJ, 239, 725

Doschek, G. A., Feldman, U., Landecker, P. B., \& McKenzie, D. L. 1981, ApJ, 249,372

Dudík, J., Janvier, M., Aulanier, G., et al. 2014, ApJ, 784, 144

Emslie, G. A., \& Alexander, D. 1987, Sol. Phys., 110, 295

Emslie, A. G., Dennis, B. R., Shih, A. Y., et al. 2012, ApJ, 759, 71

Feldman, U., Doschek, G. A., Kreplin, R. W., \& Mariska, J. T. 1980, ApJ, 241, 1175

Feng, L., Wiegelmann, T., Su, Y., et al. 2013, ApJ, 765, 37

Fisher, G. H., Canfield, R. C., \& McClymont, A. N. 1985, ApJ, 289, 414
Fletcher, L., Dennis, B. R., Hudson, H. S., et al. 2011, Space Sci. Rev., 159, 19

Forbes, T. G. 2000, J. Geophys. Res., 105, 23153

Graham, D. R., Fletcher, L., \& Hannah, I. G. 2011, A\&A, 532, A27

Harra, L. K., Sterling, A. C., Gömöry, P., \& Veronig, A. 2011, ApJ, 737, L4

Hirayama, T. 1974, Sol. Phys., 34, 323

Holman, G. D., Sui, L., Schwartz, R. A., \& Emslie, A. G. 2003, ApJ, 595, L97

Hori, K., Yokoyama, T., Kosugi, T., \& Shibata, K. 1997, ApJ, 489, 426

Hudson, H. S. 2011, Space Sci. Rev., 158, 5

Hurford, G. J., Schmahl, E. J., Schwartz, R. A., et al. 2002, Sol. Phys., 210, 61

Inoue, S., Hayashi, K., Shiota, D., Magara, T., \& Choe, G. S. 2013, ApJ, 770, 79

Inoue, S., Hayashi, K., Magara, T., Choe, G. S., \& Park, Y. D. 2015, ApJ, 803, 73

Kamio, S., Kurokawa, H., Brooks, D. H., Kitai, R., \& UeNo, S. 2005, ApJ, 625, 1027

Kamio, S., Hara, H., Watanabe, T., Fredvik, T., \& Hansteen, V. H. 2010, Sol. Phys., 266, 209

Kopp, R. A., \& Pneuman, G. W. 1976, Sol. Phys., 50, 85

Kosovichev, A. G. 2011, ApJ, 734, L15

Kosugi, T., Matsuzaki, K., Sakao, T., et al. 2007, Sol. Phys., 243, 3

Kuroda, N., Wang, H., \& Gary, D. E. 2015, ApJ, 807, 124

Landi, E., Young, P. R., Dere, K. P., Del Zanna, G., \& Mason, H. E. 2013, ApJ, 763,86

Lemen, J. R., Title, A. M., Akin, D. J., et al. 2012, Sol. Phys., 275, 17

Li, T., \& Zhang, J. 2015, ApJ, 804, L8

Lin, R. P., \& Hudson, H. S. 1976, Sol. Phys., 50, 153

Lin, R. P., Dennis, B. R., Hurford, G. J., et al. 2002, Sol. Phys., 210, 3

Litvinenko, Y. E., \& Somov, B. V. 1993, Sol. Phys., 146, 127

Long, D. M., Williams, D. R., Régnier, S., \& Harra, L. K. 2013, Sol. Phys., 288, 567

Mariska, J. T. 1992, The solar transition region (New York: Cambridge University Press)

McClements, K. G., \& Alexander, D. 1989, Sol. Phys., 123, 161

McKenzie, D. L., Broussard, R. M., Landecker, P. B., et al. 1980, ApJ, 238, L43

Milligan, R. O., \& Dennis, B. R. 2009, ApJ, 699, 968

Milligan, R. O., Gallagher, P. T., Mathioudakis, M., et al. 2006a, ApJ, 638, L117

Milligan, R. O., Gallagher, P. T., Mathioudakis, M., \& Keenan, F. P. 2006b, ApJ, 642, L169

Milligan, R. O., Kennedy, M. B., Mathioudakis, M., \& Keenan, F. P. 2012, ApJ, 755, L16

Neupert, W. M. 1968, ApJ, 153, L59

Parker, E. N. 1963, ApJS, 8, 177

Pesnell, W. D., Thompson, B. J., \& Chamberlin, P. C. 2012, Sol. Phys., 275, 3

Polito, V., Reeves, K. K., Del Zanna, G., Golub, L., \& Mason, H. E. 2015, ApJ, 803,84

Reep, J. W., Bradshaw, S. J., \& Alexander, D. 2015, ApJ, 808, 177

Scherrer, P. H., Schou, J., Bush, R. I., et al. 2012, Sol. Phys., 275, 207

Schrijver, C. J., Aulanier, G., Title, A. M., Pariat, E., \& Delannée, C. 2011, ApJ, 738,167

Sorriso-Valvo, L., De Vita, G., Kazachenko, M. D., et al. 2015, ApJ, 801, 36

Sturrock, P. A. 1968, in Structure and Development of Solar Active Regions, ed. K. O. Kiepenheuer, IAU Symp., 35, 471

Su, Y., Veronig, A. M., Holman, G. D., et al. 2013, Nature Phys., 9, 489

Sun, X., Hoeksema, J. T., Liu, Y., Chen, Q., \& Hayashi, K. 2012, ApJ, 757, 149

Teriaca, L., Falchi, A., Falciani, R., Cauzzi, G., \& Maltagliati, L. 2006, A\&A, 455, 1123

van Driel-Gesztelyi, L., Baker, D., Török, T., et al. 2014, ApJ, 788, 85

Veronig, A., Vršnak, B., Dennis, B. R., et al. 2002, A\&A, 392, 699

Veronig, A. M., Karlický, M., Vršnak, B., et al. 2006, A\&A, 446, 675

Veronig, A. M., Rybák, J., Gömöry, P., et al. 2010, ApJ, 719, 655

Veronig, A. M., Gömöry, P., Kienreich, I. W., et al. 2011, ApJ, 743, L10

Warren, H. P., \& Doschek, G. A. 2005, ApJ, 618, L157

Watanabe, T., Hara, H., Sterling, A. C., \& Harra, L. K. 2010, ApJ, 719, 213

Wiegelmann, T., Thalmann, J. K., \& Solanki, S. K. 2014, A\&ARv, 22, 78

Yokoyama, T., Akita, K., Morimoto, T., Inoue, K., \& Newmark, J. 2001, ApJ, 546, L69

Young, P. R., Doschek, G. A., \& Warren, H. P. 2011, in AAS/Solar Physics Division Abstracts 42, 2213

Young, P. R., Doschek, G. A., Warren, H. P., \& Hara, H. 2013, ApJ, 766, 127

Zarro, D. M., Canfield, R. C., Metcalf, T. R., \& Strong, K. T. 1988, ApJ, 324, 582 